\title{
Strain y Strain rate auricular izquierdo evaluado por speckle tracking está relacionado a PCR ultrasensible en adolescentes obesos
}

\author{
Luigi Gabrielli ${ }^{1}$, Pilar Arnaiz ${ }^{2}$, Mónica Acevedo ${ }^{1}$, Andrés Enriquez ${ }^{1}$, Salesa Barja ${ }^{2}$, Marlene Agloni ${ }^{2}$, \\ Samuel Córdova ${ }^{1}$, Rio Aguilar ${ }^{3}$.

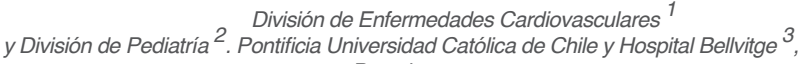 \\ Barcelona.
}

\section{Resumen:}

\section{Introducción:}

La obesidad y el síndrome metabólico están asociados a un tamaño auricular izquierdo (AI) aumentado y mayor riesgo cardiovascular. El strain y strain rate longitudinal determinado por speckle tracking son herramientas novedosas en la evaluación de la función AI.

Objetivo: evaluar el strain y strain rate AI en adolescentes obesos y su relación con biomarcadores de riesgo cardiovascular.

Métodos: se incluyeron adolescentes consecutivos con un z-índice de masa corporal $(z-I M C)>1$ en ritmo sinusal sin otra comorbilidad y un grupo control pareado por edad y sexo. Se registraron las características clínicas y ecocardiográficas con evaluación del strain AI: onda s (LASs), onda a (LASa) y strain rate AI: onda s (LASRs), onda a (LASRa) por speckle tracking. Se midió adiponectina y PCR ultrasensible.
Se utilizó t-Student, chi-cuadrado y correlación de Pearson.

Resultados: Se incluyeron 15 sujetos por grupo de edad promedio de 13 años, $47 \%$ hombres sin diferencia entre ambos grupos. Los pacientes con sobrepeso presentaron significativamente mayores niveles de PCR us respecto del grupo control, $0,5 \pm 0,1 \mathrm{mg} / \mathrm{L}$ vs $1,4 \pm 0,3, \mathrm{p}=0,04$ y menores de LASs $(44 \pm 1,8$ vs 32 $\pm 1,1 \%$; $<0,01)$, LASa $(-1,7 \pm 0,3$ vs $-0,7 \pm 0,31 / \mathrm{s}$; $\mathrm{p}<0,01)$, LASRs ( $1,7 \pm 0,07$ vs $1,3 \pm 0,03 \%$; $<<0,01)$, LASRa $(-1,8 \pm 0,1$ vs $-1,2 \pm 0,081 / \mathrm{s} ; \mathrm{p}<0,01)$. En el grupo con sobrepeso se encontró una correlación negativa significativa entre LASs, LASRs y PCR ultrasensible (R: $-0,62 ; p<0,01$ y R: $-0,58: p=0,02$ respectivamente).

Conclusiones: El strain y strain rate AI se encuentran reducidos en adolescentes obesos y estos parámetros de función AI se correlacionan con un estado inflamatorio aumentado. La importancia clínica de estos hallazgos requieren mayor investigación.

\section{Correspondencia:}

Luigi Gabrielli

Instructor Asociado

División de Enfermedades Cardiovasculares

Pontificia Universidad Católica de Chile

Igabriel@uc.cl 


\section{Speckle tracking derived left atrial strain and strain rate are related to high sensitive $\mathrm{C}$ reactive protein in obese adolescents}

Background: Obesity is an emerging problem in children worldwide and a well-known risk factor for atrial fibrillation (AF) in adulthood. There is some evidence that functional and structural changes responsible for the development of AF may arise early in life. Left atrial (LA) strain and strain rate, assessed by speckle tracking, are indices of LA function and correlate with the development of AF. Objective: To evaluate LA strain and strain rate in obese adolescents and their correlation with plasma levels of high sensitive $\mathrm{C}$ reactive protein (hs-CRP) and adiponectin, two markers of cardiovascular risk.

Methods: Fifteen obese adolescents, age $13 \pm 0.2 \mathrm{yr}$, body mass index (BMI)-z-score $1.9 \pm 0.16$ and 15 lean controls were recruited. All patients underwent transthoracic echocardiography with evaluation of LA strain and strain rate: a wave (LASa and LASRa) and s wave (LASs

\section{Introducción}

La obesidad en la infancia y adolescencia es un fenómeno creciente a nivel mundial. En Chile las cifras son preocupantes, con una prevalencia que alcanza el 20\% en escolares, según datos de la Junta Nacional de auxilio escolar y becas (JUNAEB) $)^{1-2}$. Un alto porcentaje de niños obesos continúa siéndolo en la edad adulta, con mayor riesgo cardiovascular y morbimortalidad ${ }^{3-4}$. La obesidad infantil se acompaña y favorece a otros factores de riesgo cardiovascular como hipertensión, dislipidemia y alteraciones del metabolismo de la glucosa ${ }^{5}, \mathrm{y}$ en la vida adulta la obesidad se asocia a complicaciones cardiovasculares severas como enfermedad coronaria, accidente cerebrovascular, insuficiencia cardíaca y enfermedad venosa tromboembólica ${ }^{6-7}$. A medida que aumenta el tejido adiposo, al lado del perfil metabólico alterado ocurren una variedad de adaptaciones y alteraciones en la estructura y la función cardiacas, aún en ausencia de comorbilidades ${ }^{8-9}$. En el caso de los niños que en general carecen de comorbilidades, la evidencia indica una tendencia a la disminución de la función ventricular izquierda y dilatación auricular izquierda a medida que aumenta el nivel de obesidad ${ }^{10}$. En niños obesos sin comorbilidades con una estructura y función and LASRs). Plasma levels of hs-CRP was determined. Student's $t$ test, chi-square and Pearson correlation were used for statistical analysis.

Results: Obese patients had a lower LASs $(44 \pm 1.8$ vs $32 \pm 1.1 \%, \mathrm{p}<0.01)$, LASa $(-1.7 \pm 0.3$ vs $-0.7 \pm 0.1, \mathrm{p}$ $<0.01)$, LASRs $(1.7 \pm 0.07$ vs $1.3 \pm 0.03, \mathrm{p}<0.01)$ and LASRa (- $1.8 \pm 0.1$ vs $-1.2 \pm 0.08, \mathrm{p}<0.01)$. Plasma hsCRP levels were significantly higher in the obese group and displayed a significant inverse correlation with LASs (r: $-0.62, \mathrm{p}<0.01$ ) and LASRs ( $\mathrm{r}:-0.58, \mathrm{p}=0.02$ ).

Conclusion: Obese adolescents had an impaired LA function compared to age-matched lean subjects, which correlated with an increased inflammatory state. It is likely that LA dysfunction may predispose to AF later in life. Keywords: Obesity, childhood, left atrium, speckle tracking, strain, strain rate, atrial fibrillation cardiaca normal evaluada por ecocardiografía convencional, un z-índice de masa corporal (z-IMC) elevado es un determinante independiente del aumento del tamaño de la aurícula izquierda (AI), sugiriendo que la obesidad ejerce un efecto en el tamaño de la AI a edades tempranas y potencialmente predispone a morbilidad cardiovascular más tardíamente en la vida ${ }^{11}$.

La ecocardiografía convencional y especialmente las nuevas herramientas para la evaluación de tejidos, basados en doppler tisular (TDI) y speckle tracking que permiten evaluar deformación (strain) y velocidad de deformación (strain rate) han permitido evaluar la función ventricular y auricular de forma más precisa ${ }^{12}$. Estudios en niños y adolescentes obesos han mostrado que la fracción de eyección (EF) está significativamente disminuída y el índice de performance miocárdica está significativamente alto comparados con controles normales ${ }^{13}$. La velocidad sistólica miocárdica y el strain rate están significativamente bajas, mostrando una disminución de la función sistólica ventricular 13. Además se ha demostrado una correlación negativa entre el strain rate en la base del ventrículo izquierdo (VI) y el IMC y una correlación positiva con la FE ${ }^{14}$.

Por otro lado la obesidad es un factor de riesgo independiente de fibrilación auricular (FA), aun después de 
corregir por condiciones concomitantes como la hipertensión, diabetes o infarto al miocardio ${ }^{15}$. Los mecanismos involucrados incluyen factores hemodinámicos, liberación de mediadores inflamatorios e interacciones locales directas entre los adipocitos epicárdicos y el miocardio adyacente ${ }^{16}$. La obesidad se asocia a un estado circulatorio hiperdinámico e hipervolemia, lo que conduce a presiones de llenado elevadas del VI y dilatación AI 17-18. Además, la obesidad y el síndrome metabólico pueden inducir inflamación y estrés oxidativo, que tendrían un rol en la génesis de la FA al causar remodelación eléctrica y estructural de la $\mathrm{AI}{ }^{19-21}$.

Es probable que los cambios estructurales y funcionales involucrados en la génesis de la FA comiencen a edades tempranas de la vida, sin embargo el impacto de la obesidad sobre el corazón en niños y adolescentes con sobrepeso y su eventual relación con biomarcadores no ha sido suficientemente estudiado. El strain y el strain rate longitudinal determinado por speckle tracking, son herramientas novedosas en la evaluación de la función de la AI y por esto nuestro objetivo fue evaluar, mediante estas herramientas, la condición de la AI en adolescentes obesos y la relación de estos hallazgos con biomarcadores inflamatorios de riesgo cardiovascular como es la PCR ultrasensible (PCRus).

\section{Métodos}

Estudio transversal. Se incluyeron adolescentes consecutivos con un z-IMC $>1$, en ritmo sinusal. Criterios de exclusión: edad $\geq 18$ años, evidencia al examen físico o laboratorio de resistencia a la insulina, enfermedad respiratoria crónica, neoplasia, patología congénita mayor, evidencia de daño renal (creatinina plasmática $\geq 1 \mathrm{mg} / \mathrm{dL}$ ). Se incluyó un grupo de adolescentes sanos con z-IMC $<1$ sin comorbilidad pareado por edad y sexo como grupo control. Todos los sujetos y sus padres firmaron un consentimiento informado aprobado por el comité de ética de nuestro hospital.

\section{Evaluación clínica}

Se midió peso y talla mediante balanza de palanca y estadiómetro SECA ${ }^{\circledR}$, con el niño descalzo y con ropa liviana, promediándose tres mediciones. Se calculó el IMC, expresado en percentiles y se calculó el z-score. Se definió obesidad como IMC $\geq$ percentil 95, sobrepeso: percentil 85-94, eutrofia: percentil 10-84 y bajo peso percentil $<10^{22}$. Se consideró sobrepeso y obesidad a partir de un z-score $>1$ para efectos del análisis de los grupos. Se midió la presión arterial de acuerdo a norma internacional ${ }^{23}$, con Dynamap Pro 100 , Criticon ${ }^{\circledR}$. La fase I y V de los sonidos de Korotkoff se utilizaron para la medición de la presión sistólica y diastólica. Se realizaron dos determinaciones separadas luego de 5 minutos de reposo considerándose el promedio.

Determinaciones en plama

La glicemia se midió por el método Enzimático (Hexoquinasa) / Hitachi Modular P-Roche, el colesterol Total (CT) por el método Enzimático Colorimétrico (CHODPAP) / Hitachi Modular P - Roche, el colesterol HDL (CHDL) por el método Enzimático Colorimétrico Homogéneo / Hitachi Modular P - Roche y los triglicéridos (TG) por el método Enzimático Colorimétrico con blanco-glicerol / Hitachi Modular P - Roche. El colesterol LDL (CLDL) se calculó mediante la fórmula de Friedwald $(\mathrm{LDL}=(\mathrm{CT}-\mathrm{HDL})-(\mathrm{TG} / 5))$. En todos los sujetos se realizaron mediciones plasmáticas de PCRus mediante el método nefelométrico (Dade Behring BN II Nephelometer) con un límite de detección de $0,1 \mathrm{mg} / \mathrm{L}$ ${ }^{24} \mathrm{y}$ de adiponectina mediante radioinmunoensayo (Linco Research ,Inc., St. Charles, Missouri, USA) y fue expresada en microgramos por mililitro ${ }^{24}$. La variación inter e intra ensayo fue de $8,3 \%$ y $3,4 \%$ respectivamente.

\section{Estudio ecocardiográfico.}

En cada paciente se realizó un estudio ecocardiográfico transtorácico bidimensional completo por un operador experto y ciego. Se utilizó el equipo Vivid 7 General Electric Medical Health disponible comercialmente (transductor M4S de 2.5-5 MHz). Se obtuvieron las ventanas ecocardiográficas habituales con el paciente en decúbito lateral izquierdo. Se calculó el volumen AI a partir de las visiones apicales de cuatro y dos cámaras utilizando el método biplano de discos y la masa VI mediante la fórmula de Devereux ${ }^{25}$. La función diastólica fue evaluada mediante el registro de las velocidades de influjo mitral con doppler pulsado y doppler tisular a nivel del anillo mitral medial y lateral en la visión cuatro cámaras apical.

\section{Strain y strain rate auricular izquierdo}

Para el estudio del strain y strain rate AI las imágenes fueron procesadas post adquisición con el programa Echopac7 versión 2.1.2 del 2008. Los bordes endocárdicos fueron trazados manualmente usando un puntero. El strain AI fue calculado usando el registro electrocardiográfico como referencia lo que permitió el reconocimiento del peak de la onda positiva sistólica (LASs) que corresponde a la función de conducto de la AI. De igual forma, en la curva del strain rate AI se identificó el peak 
de la onda positiva sistólica (LASRs) al inicio del sístole ventricular izquierdo y el peak de la onda negativa durante la contracción auricular (LASRa) (Figura 1). El programa divide la pared de la AI en 6 segmentos y se consideró el promedio de LASs, LASRs and LASRa para el análisis.

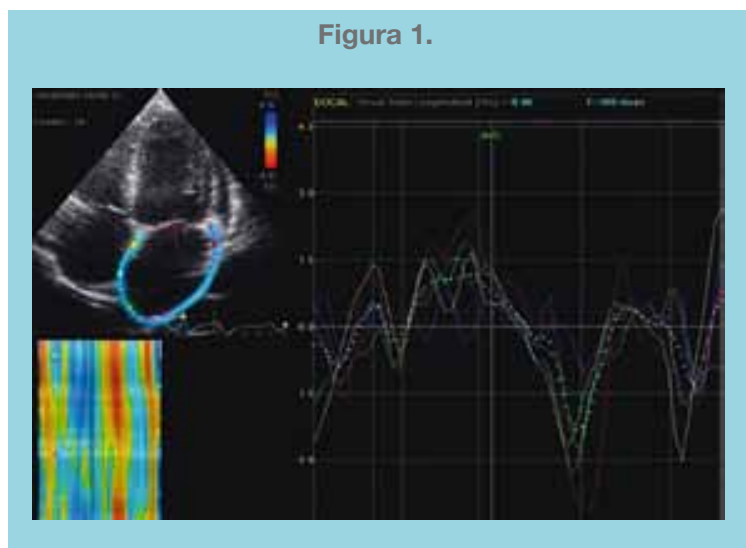

Speckle tracking bidimensional de la auricular izquierda. Curva de strain rate que muestra la onda sistólica positiva LASRs) y el peak de la onda negativa en relación a la contracción auricular (LASRa).

\section{Análisis estadístico}

Para el cálculo del tamaño muestral se utilizó el programa Power and Sample size calculations versión 2.1.31 considerando como referencia los datos de Di Salvo et al 14 . Con una potencia de $90 \%$, error alfa de $5 \%$ los grupos debían ser al menos de 10 sujetos. Las variables discretas de expresaron como porcentajes y las continuas como promedios \pm ES. Para evaluar diferencias entre grupos se utilizó chi-cuadrado o t-Student según correspondía y para la evaluación de correlaciones se utilizó el método de Pearson.

\section{Resultados}

Se incluyeron 15 sujetos por grupo de edad promedio de 13 años, 47\% hombres sin diferencia entre ambos grupos. El z-score del grupo control fue $0,1 \pm 0,05$ versus $1,9 \pm 0,16, p<0,01$ del grupo con sobrepeso. No presentaron diferencias en el estudio de la presión arterial. Los pacientes con sobrepeso presentaron un mayor diámetro indexado de la $\mathrm{AI}$ respecto del grupo control $19 \pm 0,5 \mathrm{~mm}$ versus $21 \pm 0,9, \mathrm{p}=0,03$. No presentaron otras diferencias en el estudio de dimensiones de cavidades cardíacas ni en la evaluación de la función diastólica del VI. Tabla 1.

\begin{tabular}{|c|c|c|c|}
\hline \multirow[b]{2}{*}{ Edad (años) } & Controles (15) & Sobrepeso (15) & $p$ \\
\hline & $13 \pm 0.2$ & $13 \pm 0.2$ & 0.4 \\
\hline Hombres (\%) & $47 \%$ & $47 \%$ & 0.6 \\
\hline IMC & $19 \pm 0,5$ & $29 \pm 1$ & $<0,01$ \\
\hline z IMC & $0,1 \pm 0,05$ & $1,9 \pm 0,16$ & $<0,01$ \\
\hline PAS (mmHg) & $109 \pm 1,9$ & $114 \pm 2,8$ & 0,5 \\
\hline Índice masa VI (g/m2) & $56 \pm 2,2$ & $63 \pm 2,7$ & 0,07 \\
\hline Volumen $\mathrm{Al}(\mathrm{mL} / \mathrm{m} 2)$ & $18 \pm 1$ & $19 \pm 2$ & 0,2 \\
\hline DiámetroAl (mm/m2) & $19 \pm 0.5$ & $21 \pm 0.9$ & 0.03 \\
\hline E/A & $2,2 \pm 0,2$ & $2,2 \pm 0,1$ & 0,8 \\
\hline TD (msec) & $161 \pm 6$ & $171 \pm 10$ & 0,4 \\
\hline$E / e^{\prime}$ & $7 \pm 0,3$ & $7,5 \pm 0,3$ & 0,5 \\
\hline
\end{tabular}

Abreviaciones: IMC: índice de masa corporal; PAS: presión arterial sistólica; TD: tiempo de desaceleración.

No hubo diferencias significativas en los parámetros de evaluación metabólica entre ambos grupos. Tabla 2.

\begin{tabular}{|l|l|l|l|}
\hline \multicolumn{4}{|c|}{ Tabla 2. Estudio metabólico } \\
\hline & Controles (15) & Sobrepeso & $(15) \mathrm{p}$ \\
\hline Glicemia (mg/dL) & $85 \pm 1,7$ & $83 \pm 1,4$ & 0,4 \\
\hline Colesterol total (mg/dL) & $141 \pm 5$ & $152 \pm 5$ & 0,2 \\
\hline Colesterol LDL (mg/dL) & $74 \pm 5$ & $89 \pm 4$ & 0,04 \\
Colesterol HDL (mg/dL) & $53 \pm 4$ & $44 \pm 2$ & 0,07 \\
Triglicéridos (mg/dL) & $69 \pm 14$ & $89 \pm 8$ & 0,2 \\
\hline
\end{tabular}

Abreviaciones: IMC: índice de masa corporal; PAS: presión arterial sistólica; TD: tiempo de desaceleración.

Los pacientes con sobrepeso presentaron significativamente mayores niveles de PCR us respecto del grupo control, $0,5 \pm 0,1 \mathrm{mg} / \mathrm{L}$ versus $1,4 \pm 0,3, \mathrm{p}=0,04$. No presentaron diferencias significativas en los niveles de adiponectina. Figuras 2 y 3 .

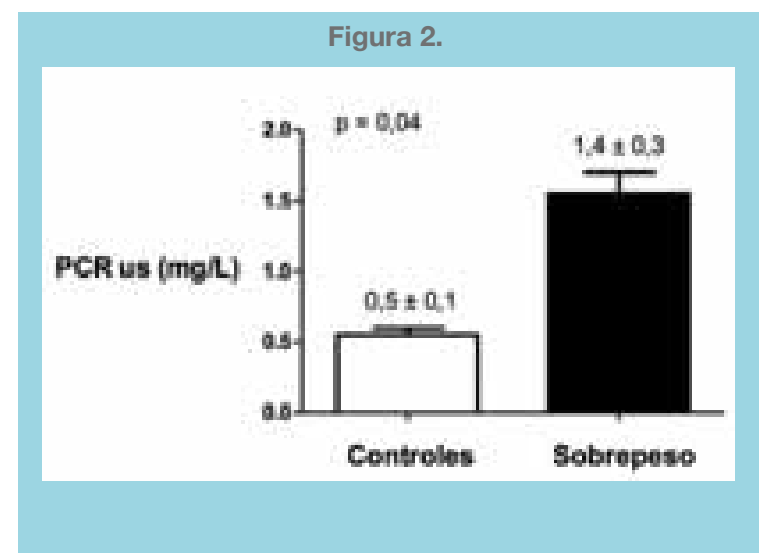

PCRus en adolescentes con sobrepeso versus sin sobrepeso. 


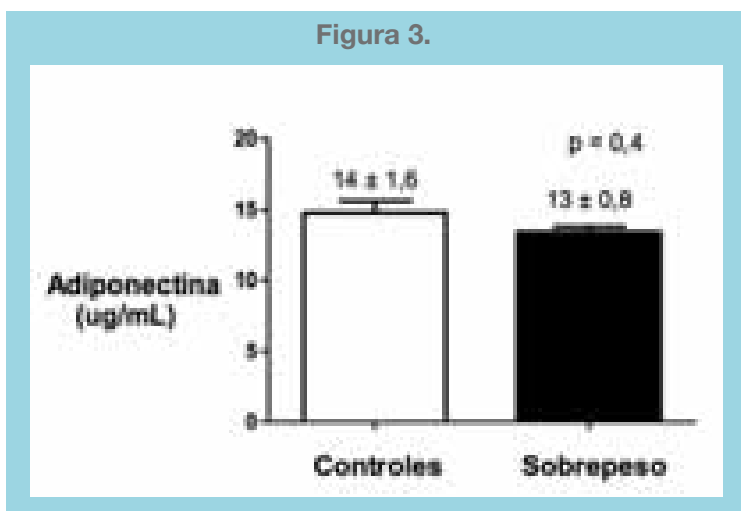

Niveles de adiponectina en adolescentes con sobrepeso versus sin sobrepeso.

En el estudio de función auricular izquierda los pacientes con sobrepeso presentaron disminución significativa de la función de conducto y contráctil tanto en el estudio con strain (Figuras 4 y 5) y strain rate (Figuras 6 y 7). Se obtuvo una correlación negativa significativa entre los niveles de PCR us y la onda s en el estudio con strain (r: $-0,62 ; \mathrm{p}<0,01)$ y strain rate $(\mathrm{r}:-0,58 ; \mathrm{p}=0,02)$ Figuras 8 y 9 . No se encontraron correlaciones significativas con la onda a ni con los niveles de adiponectina.

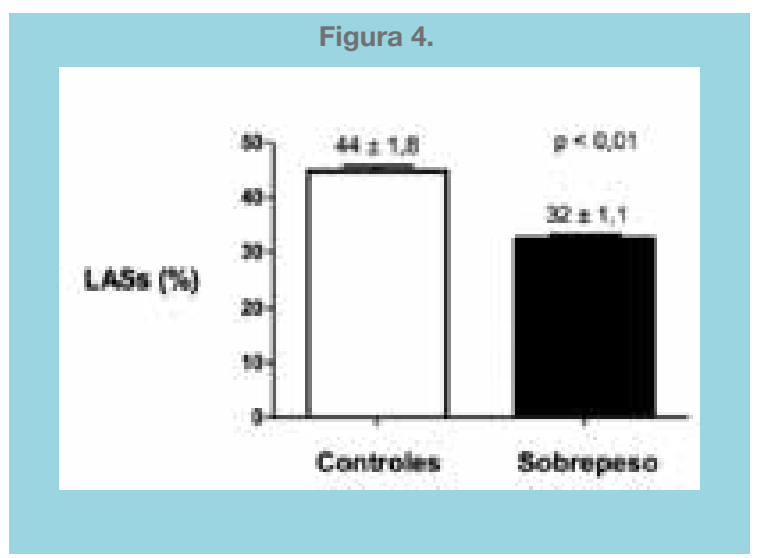

LASs en adolescentes con sobrepeso versus sin sobrepeso.

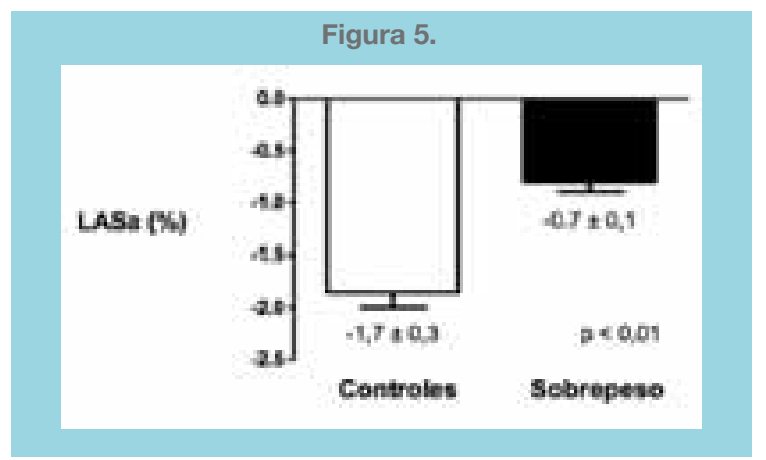

LASa en adolescentes con sobrepeso versus sin sobrepeso.

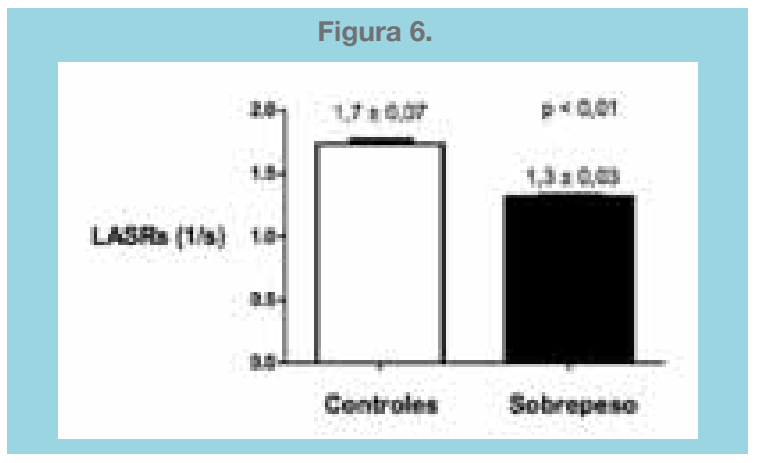

LASRs en adolescentes con sobrepeso versus sin sobrepeso.

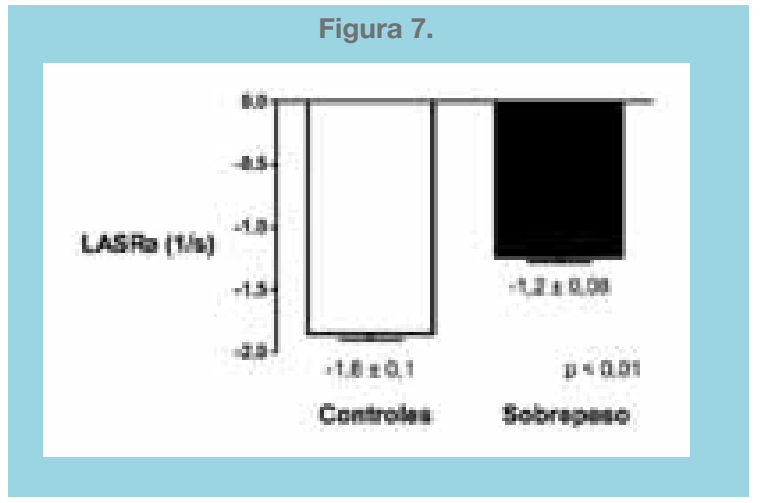

LASRa en adolescentes con sobrepeso versus sin sobrepeso.

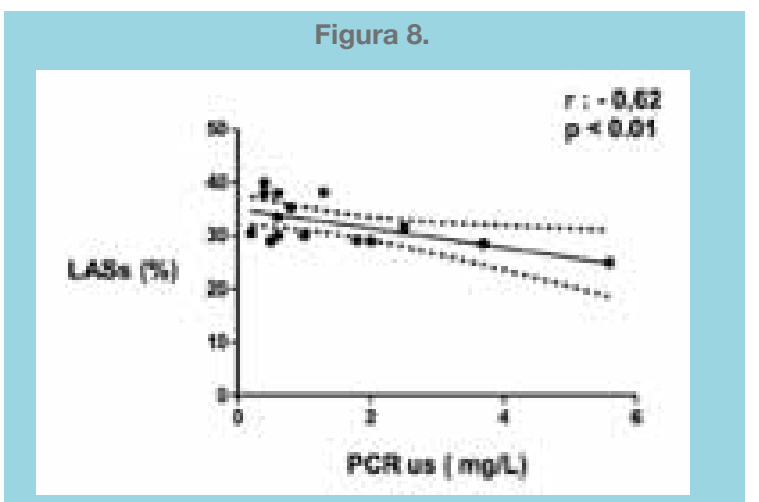

Correlación entre LASs y niveles de PCRus en adolescentes con sobrepeso.

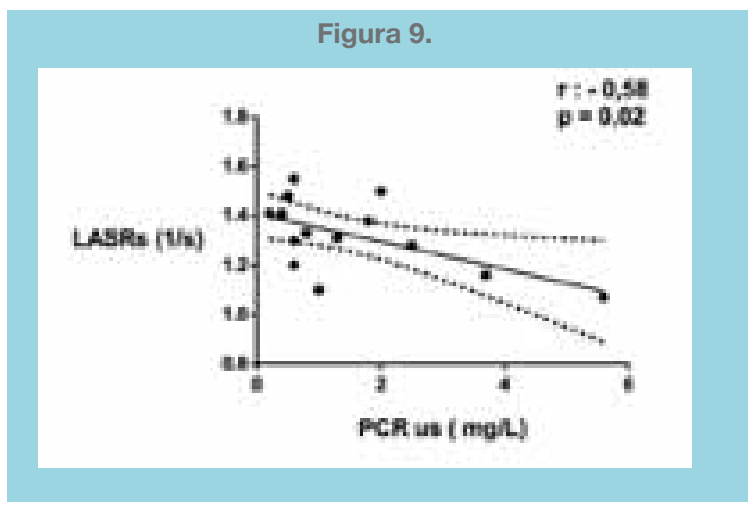

Correlación entre LASRs y niveles de PCRus en adolescentes con sobrepeso. 


\section{Discusión}

Nuestro estudio muestra que el strain y strain rate AI, dos herramientas de evaluación de la función auricular, se encuentran significativamente reducidos en adolescentes obesos. Este hallazgo complementa numerosos estudios que evidencian que la obesidad se asocia a cambios preclínicos en la estructura y función cardíaca y demuestra que estos pueden manifestarse desde etapas precoces de la vida ${ }^{26-28}$. Ambos parámetros ecocardiográficos se asocian a inflamación subclínica, determinada por PCRus en estos niños. Esta relación no había sido descrita en la literatura.

Es interesante notar que las diferencias de strain y strain rate entre pacientes obesos y controles no se acompañaron de diferencias en la función diastólica evaluada por los patrones de influjo mitral y doppler tisular. Tampoco se observó diferencias significativas en el volumen AI y sólo hubo una pequeña, aunque significativa diferencia en el diámetro auricular. Esto sugiere que las presiones de llenado elevadas del VI no constituyen el mecanismo principal en el desarrollo de disfunción auricular izquierda, o al menos no parecen jugar un rol relevante en las etapas iniciales del proceso. Dentro de los mecanismos alternativos se ha prestado importante atención al rol de la inflamación, ya que los pacientes con FA tienen niveles más elevados de PCRus 29-31 y los agentes antinflamatorios como las estatinas, los inhibidores de la enzima convertidora y los esteroides pueden prevenir la recurrencia de FA después de una cirugía cardíaca o post cardioversión ${ }^{32-34}$. En los individuos obesos existe evidencia de que el aumento del tejido adiposo se asocia a un estado inflamatorio de baja cuantía, pero crónico, evidenciado por un aumento de IL-6 y PCRus ${ }^{35-37}$. Se postula que este estado inflamatorio sistémico participa en la patogenia de la enfermedad aterosclerótica prematura y es posible que pueda jugar un rol en el remodelado auricular y en el desarrollo de FA ${ }^{31,36}$. La grasa epicárdica es una fuente importante de mediadores inflamatorios y adipocitoquinas, que pueden producir directamente remodelado eléctrico y estructural de la aurícula ${ }^{37}$. Malavazos et al., demostraron que los niveles de proteína quimiotáctica de los monocitos 1 y del complejo IL-6/receptor soluble de IL-6 se correlacionan estrechamente con el espesor de la grasa epicárdica ${ }^{38}$ y Kremen et al., detectaron altas concentraciones de citokinas inflamatorias en la grasa epicárdica de pacientes sometidos a cirugía cardíaca ${ }^{39}$. Dado que la grasa epicárdica está en contacto directo con la AI y las venas pulmonares, su rol puede ser clave en la iniciación de la FA. En concordancia con estos datos, nuestro estudio demostró que el deterioro de la función AI, manifestado por una disminución del strain y starin rate $\mathrm{AI}$, en adolescentes obesos se correlaciona con un estado inflamatorio aumentado, expresado por niveles significativamente más elevados de PCRus.

No se encontró asociación entre el strain o strain rate auricular en nuestros pacientes y los niveles de adiponectina. Esta es una hormona derivada del tejido adiposo con propiedades antidiabéticas, antiaterogénicas y antiinflamatorias y se le ha atribuido un rol protector contra la fibrosis y remodelación miocárdica patológica. Los niveles plasmáticos de adiponectina se encuentran disminuidos en los individuos obesos y se postula que este es un factor que contribuye al remodelado auricular ${ }^{40,41}$. El hecho de no encontrar una diferencia significativa en nuestro grupo de estudio, pudiera deberse al hecho que la adiponectina en los niños obesos hombres pareciera empezar a disminuir recién después de la pubertad, como fue publicado por nuestro mismo grupo recientemente ${ }^{24}$. Por lo mismo, se requieren futuros estudios que permitan aclarar la eventual relación de la adiponectina, la función AI y el desarrollo de la FA en pacientes obesos.

Dentro de las limitaciones de este estudio creemos que habría sido valioso el incorporar marcadores de fibrosis miocárdica como el telopéptido C-terminal del colágeno tipo I, un marcador de la degradación del colágeno tipo I, o el péptido N-terminal del procolágeno tipo III, un marcador de síntesis del colágeno tipo III.

En conclusión, hemos demostrado no sólo alteraciones estructurales y funcionales en la AI de niños obesos, sino que también demostrado asociación a alteraciones de tipo bioquímico. La importancia de estos hallazgos radica en que la obesidad es un factor de riesgo totalmente modificable. Como vemos, el sustrato responsable de su génesis comienza a establecerse precozmente durante la vida del individuo, lo que refuerza la necesidad de establecer estrategias eficaces y agresivas para prevenir la obesidad infantil en nuestro país. La corrección de estas alteraciones estructurales y funcionales que se producen en el corazón del niño y adolescente obeso, podrían traducirse en un impacto importante en la incidencia futura de enfermedades tan deletéreas como la FA del adulto. 


\section{Referencias:}

1. ARNAIZ P, ACEVEDO M. Arterioesclerosis subclínica, factores de riesgo cardiovascular clásicos y emergentes en niños obesos chilenos. Rev Chil Pediatr 2007;78: 135-142.

2. Estadística Junta Nacional de auxilio escolar y becas (JUNAEB) 2006.

3. MUST A, JACQUES PF, DALLAL GE, BAJEMA CJ, DIETZ WH. Long term morbidity and mortality of overweight adolescents: a follow up of the Harvard Growth Study of 1922 to 1935. N Engl J Med 1992; 327:1350-5.

4. FREEDMAN DS, KHAN LK, DIETZ WH, SRINIVASAN SR, BERENSON GS. Relationship of childhood obesity to coronary Heart disease risk factors in adulthood: the Bogalusa Heart Study. Pediatrics 2001; 108: 712-8.

5. COOK S, WEITZMAN M, AUINGUER P, NGUYEN M, DIETZ WH. Prevalence of a metabolic syndrome phenotype in adolescents: findings from the third National Health and Nutrition Examination Survey, 1988-1994. Arch Pediatr Adolesc Med 2003; 157: 821-7.

6. HUBERT HB, FEINLEIB M, MCNAMARA PM. Obesity as an independent risk factor for cardiovascular disease: a 26-year follow-up of participants in the Framingham Heart Study. Circulation 1983; 67: 968-77.

7. KENCHAIAH S, EVANS JC, LEVY D. Obesity and the risk of heart failure. N Engl J Med. 2002; 347: 305-13.

8. GATES P, GENTILE C, SEALS D, CHRISTOU D. Adiposity contributes to differences in left ventricular structure and diastolic function with age in healthy men. J Clin Endocrinol and Metab 2003; 88: 4884-4890.

9. ROWLAND TW. Effect of obesity on cardiac function in children and adolescents: a review. J Sports Science and Med 2007; 6: 319-326.

10. CHINALI M, DE SIMONE G, ROMAN M, LEE E, BEST L, HOWARD B, et al. Impact of obesity on cardiac geometry and function in a population of adolescents The Strong Herat Study. J Am Coll Cardiol 2003; 47: 2267-73.

11. AYER JG, SHOLLER GF, CELERMAJER DS. Left atrial size increases with body mass index in children. Int J Cardiol. 2010; 141: $61-7$

12. SARAIVA RM, DEMIRKOL S, BUAKHAMSRI A, GREENBERG N, POPOVIĆ ZB, THOMAS JD, et al. Left atrial strain measured by two-dimensional speckle tracking represents a new tool to evaluate left atrial function. J Am Soc Echocardiogr 2010; 23: $172-80$.

13. HEO JA, HONG YM. Left Ventricular systolic function by tissue doppler imagin and strain rate imaging in obese adolescents. J Korean Pediatr Cardiol Soc 2005; 9: 145-154.
14. DI SALVO G, PACILEO G, DEL GIUDICE EM, NATALE F, LIMONGELLI G, VERRENGIA M, et al. Abnormal myocardial deformation properties in obese, non-hypertensive children: an ambulatory blood pressure monitoring, standard echocardiographic, and strain rate imagin study. Eur Heart J. 2006; 27: 2689-95.

15. WANG TJ,PARISE H,LEVY D. Obesity and the risk of newonset atrial fibrillation. JAMA 2004; 292:2471-7.

16. LIN YK, CHEN YJ, CHEN SA. Potential atrial arrhythmogenicity of adipocytes: implications for the genesis of atrial fibrillation. Med Hypotheses 2010;74:1026-9.

17. PASCUAL DA, SORIAF, VICENTE T. Effects of isolated obesity on systolic and diastolic left ventricle function. Heart 2003; 89:1152-6.

18. PRITCHETT AM, MAHONEY DW, JACOBSEN SJ, RODEHEFFER RJ, KARON BL, REDFIELD MM. Diastolic dysfunction and left atrial volume: a population-based study. J Am Coll Cardiol 2005; 45: 87-92.

19. ENGELMANN MD, SVENDSEN JH. Inflammation in the genesis and perpetuation of atrial fibrillation. Eur Heart J 2005; 26 : 2083-92.

20. BOOS CJ, ANDERSON RA, LIP GY. Is atrial fibrillation an inflammatory disorder? Eur Heart J 2006; 27: 136-49.

21. FORD ES, MOKDAD AH, GILES WH, BROWN DW. The metabolic syndrome and antioxidant concentrations: findings from the Third National Health and Nutrition Examination Survey. Diabetes 2003; 52: 2346-52.

22. CDC.gov. 2000 CDC Growth Charts. Disponible en: http:// www.cdc.gov/growthcharts/

23. National High Blood Pressure Education Program. Working Group on High Blood Pressure in Children and Adolescents. The Fourth report on the Diagnosis, Evaluation, and Treatment of High Blood Pressure in Children and Adolescents. Pediatrics 2004; 114: 555-576.

24. ARNAIZ P, ACEVEDO M, BARJA S, AGLONY M, GUZMÁN B, CASSIS B. Adiponectin levels, cardiometabolic risk factors and markers of subclinical atherosclerosis in children. Int J Cardiol 2010; 138:138-44.

25. LANG RM, BIERIG M, DEVEREUX RB, FLACHSKAMPF FA, FOSTER E, PELLIKKA PA. Recommendations for chamber quantification. Eur J Echocardiogr 2006; 7: 79-108.

26. SUNG JK, KIM JY. Obesity and preclinical changes of cardiac geometry and function. Korean Circ J. 2010 Feb;40:55-61.

27. AYER JG, SHOLLER GF, CELERMAJER DS. Left atrial size increases with body mass index in children. Int $\mathrm{J}$ Cardiol 2010;141:61-7.

28. URBINA EM, GIDDING SS, BAO W, PICKOFF AS, BERDUSIS K, BERENSON GS. Effect of body size, ponderosity, and blood pressure on left ventricular growth in children and 
young adults in the Bogalusa Heart Study. Circulation.1995; $91: 2400-2406$

29. CHUNG MK, MARTIN DO, SPRECHER D. C-reactive protein elevation in patients with atrial arrhythmias: inflammatory mechanisms and persistence of atrial fibrillation. Circulation 2001;104: 2886-91.

30. MCCABE JM, SMITH LM, TSENG ZH. Protracted CRP elevation after atrial fibrillation ablation. Pacing Clin Electrophysiol 2008; 31: 1146-51.

31. ACEVEDO M, CORBALÁN R, BRAUN S, PEREIRAJ, NAVARRETE C, GONZALEZ I. C-reactive protein and atrial fibrillation: "evidence for the presence of inflammation in the perpetuation of the arrhythmia".Int J Cardiol. 2006 Apr 14; 108: 326-31.

32. PATTI G, CHELLO M, CANDURA D. Randomized trial of atorvastatin for reduction of postoperative atrial fibrillation in patients undergoing cardiac surgery: results of the ARMYDA-3 (Atorvastatin for Reduction of Myocardial Dysrhythmia After cardiac surgery) study. Circulation 2006; 114: 1455-61.

33. UENG KC, TSAI TP, YU WC. Use of enalapril to facilitate sinus rhythm maintenance after external cardioversion of longstanding persistent atrial fibrillation. Results of a prospective and controlled study. Eur Heart J 2003; 24: 2090-8.

34. GOLDSTEIN RN, RYU K, KHRESTIAN C, VAN WAGONER

DR, WALDO AL. Prednisone prevents inducible atrial flutter in the canine sterile pericarditis model. J Cardiovasc Electrophysiol 2007; 19: 74-81.
35. FORD DS. C reactive protein concentration and cardiovascular disease risk factors in children. Circulation 2003; 108: 1053-8.

36. FONTANA L, EAGON JC, TRUJILLO ME. Visceral fat adipokine secretion is associated with systemic inflammation in obese subjects. Diabetes 2007; 56: 1010-3.

37. ACEVEDO M, ARNAIZ P, BARJA S, BAMBS C, BERRIOS X, GUZMAN B. Relationship of C-reactive protein to adiposity, cardiovascular risk factors and subclinical atherosclerosis in healthy children. Rev Esp Cardiol. 2007;60:1051-8

38. MALAVAZOS AE, ERMETICI F, COMAN C, CORSI MM, MORRICONE L, AMBROSI B. Influence of epicardial adipose tissue and adipocytokine levels on cardiac abnormalities in visceral obesity. Int J Cardiol 2007;121:132-4.

39. KREMEN J, DOLINKOVA M, KRAJICKOVA. Increased subcutaneous and epicardial adipose tissue production of proinflammatory cytokines in cardiac surgery patients: possible role in postoperative insulin resistance. J Clin Endocrinol Metab 2006; 91:4620-7.

40. SHIMANO M,SHIBATAR, TSUJI Y, KAMIYAH, UCHIKAWA T, HARATA $S$, et al. Circulating adiponectin levels in patients with atrial fibrillation. Circ J. 2008; 72: 1120-4.

41. YBARRA J, RESMINI E, PLANAS F, NAVARRO-LÓPEZ F, WEBB S, POU JM, et al. Relationship between adiponectin and left atrium size in uncomplicated obese patients: adiponectin, a link between fat and heart. Obes Surg. 2009; 19: 1324-32. 